\title{
Electrons and Water Molecules Form a Pulsating Cluster
}

\author{
In water, single electrons can cluster with water molecules to form a \\ quasiparticle that oscillates in size, a behavior that could influence the \\ equilibration speed of chemical reactions in the system.
}

\section{By Sophia Chen}

W

hen a free electron in water interacts

with neighboring water molecules, it can form a quasiparticle known as a "solvated" electron. How these solvated electrons behave provides fundamental insights for charge transport and chemical reactions. Now, Michael Wörner of the Max Born Institute in Germany and colleagues have observed solvated electrons in water inducing previously unseen terahertz-scale oscillations in the water's polarization [1]. These oscillations may play an important role in how a chemical reaction approaches equilibrium.

To produce solvated electrons, the researchers applied pulses of terahertz and near-infrared radiation to a 50- $\mu$ m-wide jet of water. The radiation stripped some water molecules of electrons. These electrons moved a short distance before localizing because of interactions with other water molecules. To understand the liquid's properties when that happened, the researchers used terahertz light to monitor

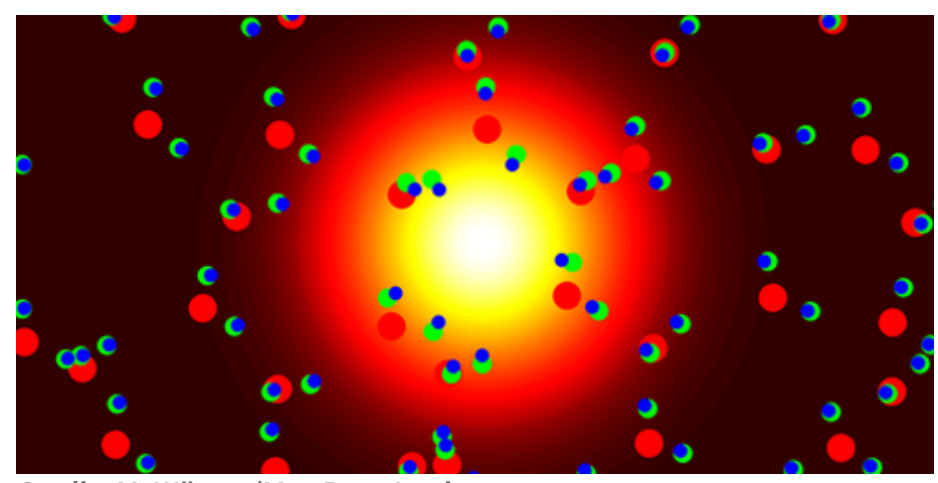

Credit: M. Wörner/Max Born Institute polarizability-which relates to how easily the liquid developed a dipole moment in response to an electric field.

The researchers observed the liquid's polarization oscillate in magnitude. The oscillations lasted tens of picoseconds and had a frequency of between 0.2 to $1.5 \mathrm{THz}$, with the higher frequencies occurring for higher electron concentrations. Using a previously developed theoretical model, Wörner and colleagues determined that this oscillatory response arose from the quantized motion of the solvated electrons.

The researchers explained the generation of oscillations as follows: When an electron initially detaches from its water molecule, the electron has a high kinetic energy. That energy decreases as the electron interacts with other water molecules. After losing a certain amount of energy, the electron interacts with nearby water molecules, whose dipole moments point toward the electron, forming a solvated electron. This quasiparticle oscillates in size as it gains the electron's remaining energy, leading to the terahertz-duration oscillations that the team measured.

Sophia Chen is a freelance science writer based in Columbus, Ohio.

\section{REFERENCES}

1. A. Ghalgaoui et al., "Terahertz polaron oscillations of electrons solvated in liquid water," Phys. Rev. Lett. 126, 097401 (2021). 